\title{
ON MAGNETICALLY COUPLED COILS PARAMETER CALCULATION
}

\author{
LAVINIA MARIA BOBARU, MARILENA STĂNCULESCU, D. NICULAE, M. IORDACHE \\ University Politehnica of Bucharest, Spl. Independentei, ROMANIA, Electrical Engineering Faculty / Dept. of \\ Electrical Engineering, \\ E-mail: lavinia.bobaru@upb.ro, marilena.stanculescu@upb.ro
}

\begin{abstract}
ANSYS Q3D Extractor tool (AQ3DE), using the quasi-static solvers (method of moments, integral equations and FEMs), is very efficient for computing and simulating two- and three-dimensional electromagnetic field. This simulation is used to extract parameters of interest, such as: resistance $(R)$, conductance $(G)$, self-inductance $(L)$, mutual inductance $(M)$ and capacitance $(C)$. These parameters can be further used to optimize and to obtain automatically the design of a wireless power transfer systems (WPTS). The resulting matrices allow the generation of new matrices for any selected parameters. To complete the analysis, the model which is computed will be exported as an equivalent circuit. In this paper, starting from a given WPTS, using AQ3DE tool, the parameters of interest $(R, G, L, M, C)$ were automatically calculated, and the SPICE equivalent circuit was obtained. The analysis was performed for two different distances between the emitter and the receiver. The electrical circuit theory was used for the system analysis of magnetically coupled coil (MCC) system. Starting from the parameters extracted by the AQ3DE tool, the performance of the WPTS has been analysed both in time and frequency domain.
\end{abstract}

Keywords: ANSYS DesignerSI, AC/DC analysis, SPICE, magnetically coupled coils, optimization, circuit simulation

\section{INTRODUCTION}

For engineering design and for power electronics domain, one of the most suitable software is ANSYS Q3D Extractor software (AQ3DE) [1]. This software allows the extraction of two- and three-dimensional parameters.

AQ3DE, using the quasi-static solvers (finite element method), is very efficient for computing and simulating two- and three-dimensional electromagnetic field [2]. This simulation is used to extract parameters of interest, such as: resistance $(R)$, conductance $(G)$, self-inductance $(L)$, mutual inductance (M) and capacitance (C). These parameters can be further used to optimize and to obtain automatically the design of a wireless power transfer systems (WPTS). This simulation is used to extract the resistance, conductance, self-inductance, mutual inductance and capacitance $(R, G, L, M, C)$ parameters for an optimal and automatic design of a WPTS [3-6]. In this way a list of the electronic components in a circuit and a list of the nodes (netlist) is automatically generated. The generation of the netlist can be done in various formats such as: ANSYS Simplorer format (.sml) and SPICE format (.cir). The solver will output the matrices of interest $(\boldsymbol{C}, \boldsymbol{G}, \boldsymbol{R}, \boldsymbol{L})$ and voltage distributions and current distributions. To accelerate the solution, AQ3DE uses the method called fast multipole method (FMM) [1], [7-9].
For higher electromagnetic field operating frequencies, when the radiation is important, AQ3DE uses the fullwave solvers to generate the $\mathrm{S}, \mathrm{Y}$, and $\mathrm{Z}$ parameters and fields [10-13]. AQ3DET can also generate the parameters $\mathrm{S}$ which are further exported in Touchstone format (i.e. a text file which contain the parameter data of an n-port network). The parameters which depend on high frequencies can be simulated using products such as: ANSYS Designer SI or ANSYS Simplorer [1, 2].

DesignerSI is a very accurate design platform. It puts together the circuit simulation and analysis with the analysis of the electromagnetic field. With this powerful tool, the simulation of the integrity of the signal can be handled using different approaches and methods. More, the methods contain optimization algorithms, tuning algorithms, design of experiments (DOE) algorithms, etc. Using AQ3DE solver, a complete package of structures can be characterized and the values of the $R, L, C, G$ (Resistance, Inductivity, Capacity and Conductance) matrices are automatically calculated or distributed. The generation of $\boldsymbol{G}$ and $\boldsymbol{C}$ matrices are performed using the static electromagnetic field analyses [14-18]. To generate $\boldsymbol{R}$ and $\boldsymbol{L}$ there are used ac and dc analyses. Finally, the software generates a SPICE file that can be exported for analysis with a circuit simulator.

For the given workbench, by analyzing the results, one can choose the most suitable solution (optimal).

In this paper, starting from a given WPTS, using AQ3DE tool, the parameters of interest $(\mathrm{R}, \mathrm{G}, \mathrm{L}, \mathrm{M}, \mathrm{C})$ were automatically calculated, and the SPICE equivalent circuit was obtained. The analysis was performed for two different distances between the emitter and the receiver. The electrical circuit theory was used for the system analysis of magnetically coupled coil (MCC) system. Starting from the parameters extracted by the AQ3DE tool, the performance of the WPTS has been analyzed both in time and frequency domain

\section{DESCRIPTION OF ANSOFT Q3D EXTRACTOR Q3D (Q2D)}

2D and 3D interconnected structures can be fully characterized from electrical and magnetical point of view by using the dedicated software AQ3DE $[1,2]$. By using AQ3DE, one obtains the parameters:

- Capacity matrices $(\mathbf{C})$ in static regime; 
- Self and mutual inductivities and resistances matrices $(\underline{\mathbf{L}, \mathbf{M}, \mathbf{R}})$ in dc analysis;

- Resistances, self and mutual inductivities and resistances matrices $(\boldsymbol{L}, \boldsymbol{M}, \boldsymbol{R})$ for high frequency ac analysis.

The procedure for computing the parameters of magnetically coupled coils (MCC), involves the following preliminary steps:

- $\quad$ select the analysis to be performed;

- obtain the model;

- $\quad$ provide the material properties;

- identification of the conductive elements;

- $\quad$ specification of the excitation source.

The parameters needed for the circuit are automated generated. The system automatically generates the necessary parameters of the circuit. The MCC parameters identification [6], [11], [15] used in WPTS, is based on Maxwell's equations (general and local forms of the laws).

As a case study, we consider a WPTS, which consist of two coils, magnetically coupled, helix shape (Figure 1).

The two coils form a WPTS. Denote by Tx - the transmitter (transmitting coil) and by $\mathrm{Rx}$ - the receiver (receiving coil). Both coils are identical with given parameters:

- helix coil: rectangular section: $1.71 \mathrm{~mm}$ x 3.20 $\mathrm{mm}$;

- $\quad$ pitch (distance between two consecutive turns): $2.2 \mathrm{~mm}$;

- 2 layers each having 8 turns in series connection; distance between layers: $2 \mathrm{~mm}$.

Between the two coils the distance can be changed (the results were analysed for $\mathrm{z}=20.2 \mathrm{~mm}$, respectively $\mathrm{z}=70$ $\mathrm{mm})$.

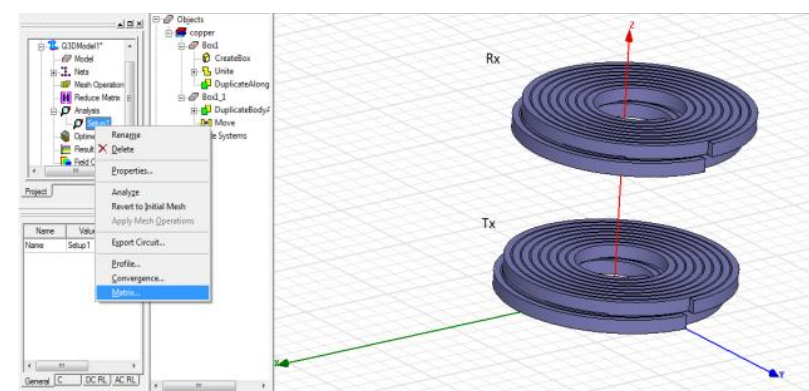

Figure 1. Two magnetically coupled coils, helix shape.

\section{MODELLING PROCESS ALGORITHM}

The parameters of interest, $\boldsymbol{R}, \boldsymbol{G}, \boldsymbol{L}, \boldsymbol{C}$ are computed using AQ3DE. The next steps are performed by the software:

- The structure is divided in finite elements network;

- Inside the structure, the electromagnetic field is computed, function of the selected type of analyses (a source terminal or a single conductor is excited at a given moment);

- The parameters $\boldsymbol{R}, \boldsymbol{L}, \boldsymbol{G}$ and $\boldsymbol{C}$ are computed function of the field's values - computed above.

For any selected parameters, new matrices can be obtained based on the previously resulting matrices. For further analysis, the results will be exported to an equivalent circuit (Figure 2).

The matrix's inputs are computed by the solver. The conductive elements influence the calculation of the parameter's matrices.

After the solution of the field is computed and the corresponding matrices are completely calculated, AQ3DE is performing the analysis of the error. This analysis takes place for any element belonging to a loop of the network.

To obtain an accurate solution, the computation is adapted to be performed on smaller elements.

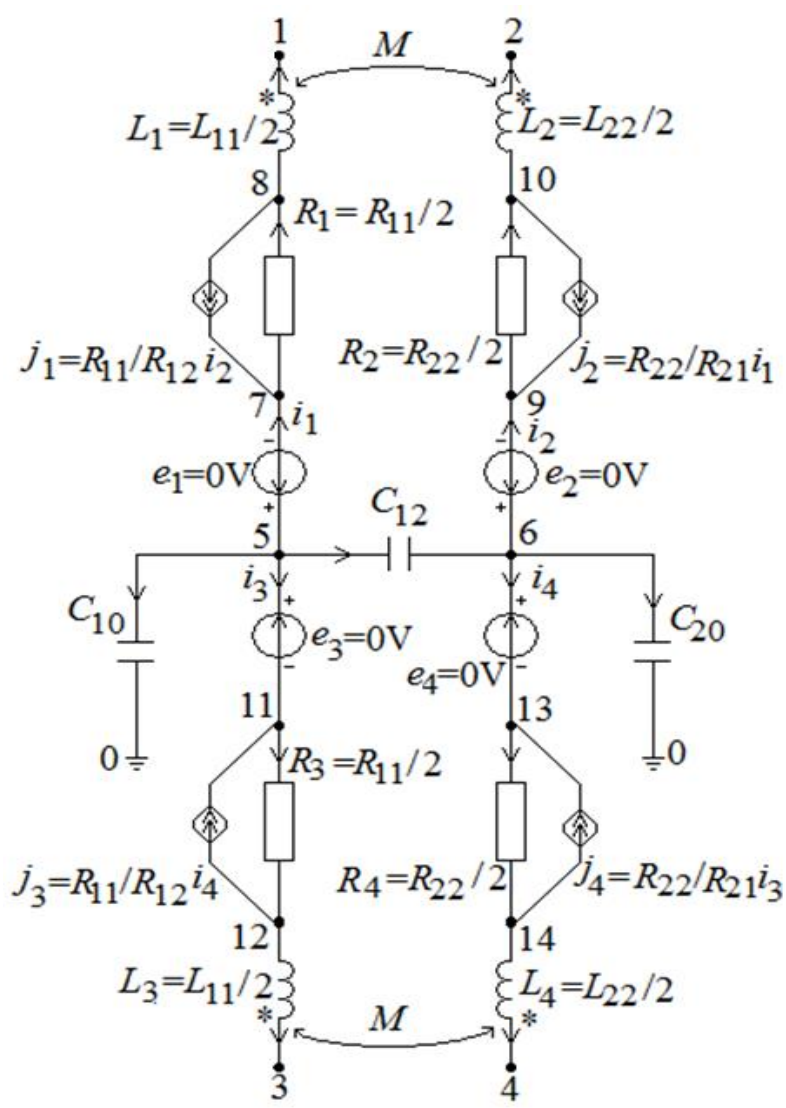

Figure 2. SPICE equivalent circuit obtained by the Q3D EXTRACTOR simulator.

\section{NUMERICAL RESULTS}

The obtained numerical results for the proposed case study (Figure 1), are presented in Table 1. The values are given in Ansys format.

Table 1. Parameters obtained with the AQ3DE for $\mathbf{f}$ $=20 \mathbf{~ k H z}$ 


\begin{tabular}{|c|c|c|c|c|}
\hline $\begin{array}{c}\mathrm{h}[\mathrm{mm}] \\
\text { (distance } \\
\text { between } \\
\text { the coils) }\end{array}$ & $\begin{array}{c}\mathrm{C}[\mathrm{pF}] \\
\text { (capacity } \\
\text { matrix) }\end{array}$ & $\begin{array}{c}\mathrm{R}[\mathrm{m} \Omega] \\
\text { (matrix of } \\
\text { resistances) }\end{array}$ & $\begin{array}{c}\mathrm{L}[\mathrm{nH}] \\
\text { (matrix of } \\
\text { inductivities }\end{array}$ & $\mathrm{k}=\mathrm{M} / \sqrt{L 1} * L 2$ \\
\hline 20.2 & $\left(\begin{array}{rr}2.9011 & -1.2613 \\
-1.2613 & 2.9022\end{array}\right)$ & $\left(\begin{array}{cc}3.3633 & 0.26225 \\
0.26225 & 3.4022\end{array}\right)$ & $\left(\begin{array}{ll}44.928 & 2.5835 \\
2.5835 & 44.85\end{array}\right)$ & $\mathbf{0 . 0 5 7 5 7}$ \\
\hline 70 & $\left(\begin{array}{rr}2.4635 & -0.5544 \\
-0.5544 & 2.4641\end{array}\right)$ & $\left(\begin{array}{ll}3.0806 & 0.0388 \\
0.0388 & 3.0791\end{array}\right)$ & $\left(\begin{array}{ll}45.423 & 0.4072 \\
0.4072 & 45.408\end{array}\right)$ & 0.00236 \\
\hline
\end{tabular}

In SPICE format the capacities matrices differ from one in Ansys format.

To obtain the SPICE format, we proceed as follows:

$$
\begin{aligned}
& \text { C11_Spice }=\text { C11_Ansys }+ \text { C12_Ansys } \\
& \text { C12_Spice }=- \text { C12_Ansys } \\
& \text { C12=C21 }
\end{aligned}
$$

Taking this into account, results the values for the capacities the SPICE format:

$$
\left(\begin{array}{ll}
1.6398 & 1.2613 \\
1.2613 & 1.6409
\end{array}\right)\left(\begin{array}{ll}
1.90901 & 0.55449 \\
0.55449 & 1.90961
\end{array}\right)
$$

In Figure 3 is shown the magnetic field strength $[\mathrm{A} / \mathrm{m}]$ for $\mathrm{z}=20.2 \mathrm{~mm}$. For $\mathrm{z}=70 \mathrm{~mm}$, the magnetic field strength $[\mathrm{A} / \mathrm{m}]$ is given in Figure 4.

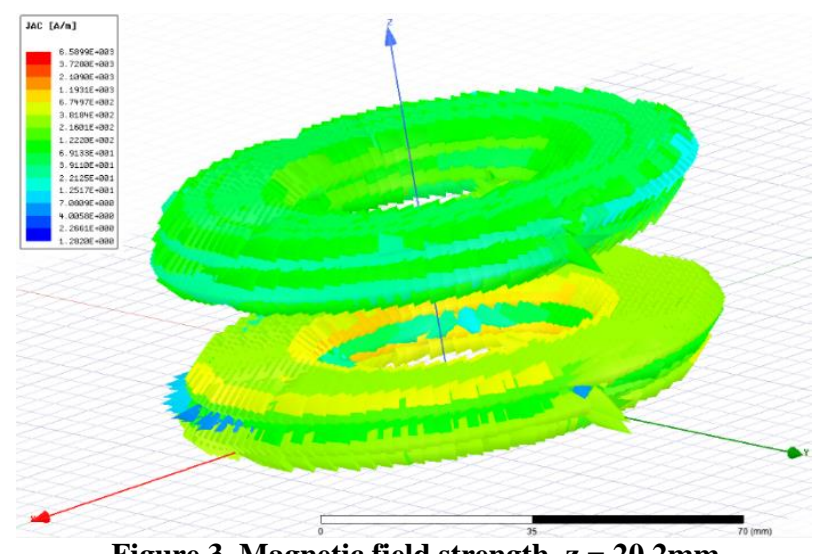

Figure 3. Magnetic field strength, $z=20.2 \mathrm{~mm}$

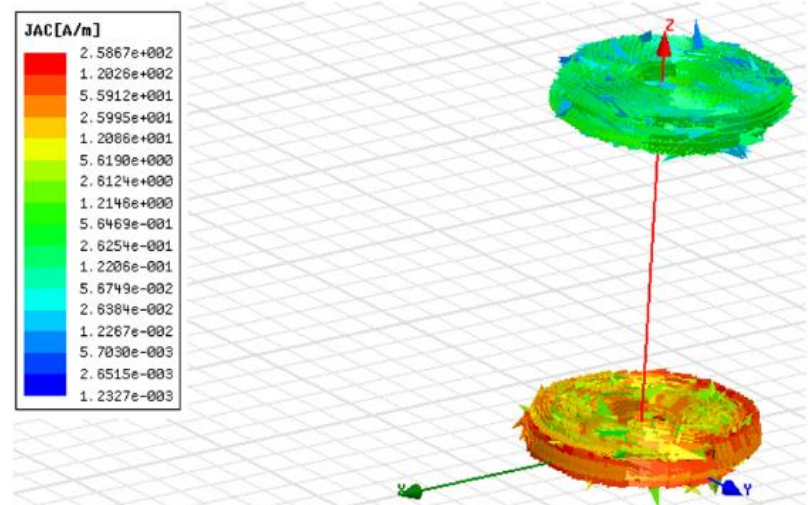

Figure 4. Magnetic field strength, $\mathrm{z}=70 \mathrm{~mm}$.
In Figure 5, a there is presented the topology of two MCC with series connection, and in Figure $5, \mathrm{~b}$ is drawn the operational diagram of the system.

By considering the initial conditions, null, one can replace the magnetic coupling with two current-controlled voltage sources (CCVT)

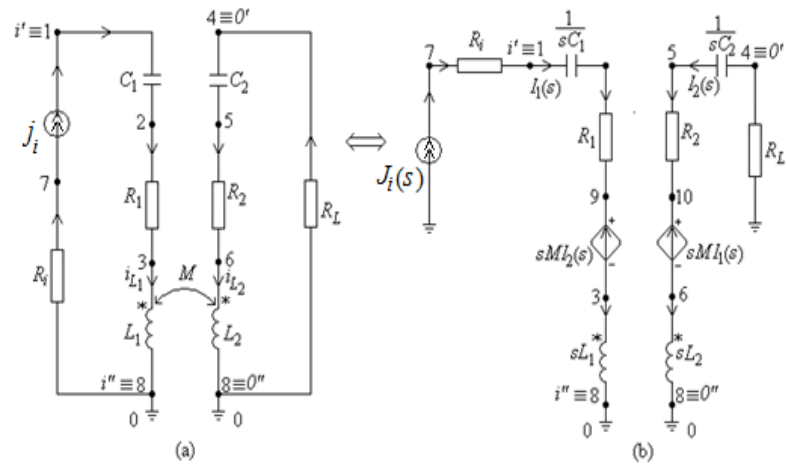

Figure 5. a) Topology of two magnetically coupled coils system with series connection; b) The operational diagram of the system.

Using AQ3DE, one obtains the parameters corresponding to the MCC. The values are listed below:

$$
\begin{aligned}
& L_{1}=63.76 \mu \mathrm{H}, L_{2}=32.6 \mu \mathrm{H}, M=1.5957 \mu \mathrm{H}, \\
& k=0.35, C_{1}=0.15844 \mu \mathrm{F}, C_{2}=0.31 \mu \mathrm{F}, \\
& R_{1}=0.0925 \Omega, R_{2}=0.0545 \Omega, J_{\mathrm{i}}=20 \mathrm{~A}, \\
& R_{\mathrm{i}}=1.5 \Omega, R_{\mathrm{L}}=6.0 \Omega, f_{0}=50.1 \mathrm{kHz}, \\
& \omega_{0}=3.1463 \mathrm{e} 05 \mathrm{rad} / \mathrm{s}, \\
& Q_{1}=\omega_{0} L_{1} /\left(R_{\mathrm{i}}+R_{1}\right)=12,597 \\
& Q_{2}=\omega_{0} L_{2} / R_{2}=188.1995 .
\end{aligned}
$$

Analyzing the circuits in Figure 5, a and b in time domain for $i=20 \sin (2 \pi \mathrm{ft})[\mathrm{A}]$, we obtain the results presented in Figure 6 - 8 .

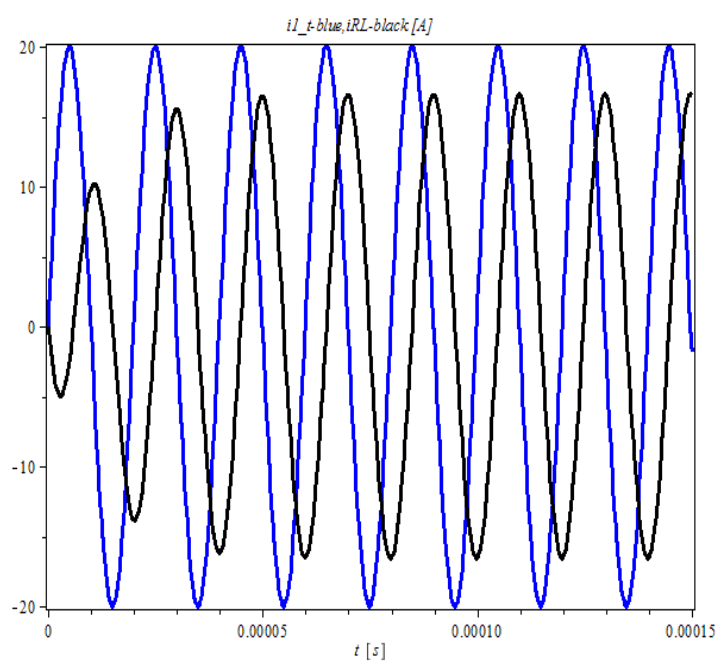

Figure 6. $i_{1} \mathrm{t}$ and $i_{R_{1} \_t}$ vs. time. 


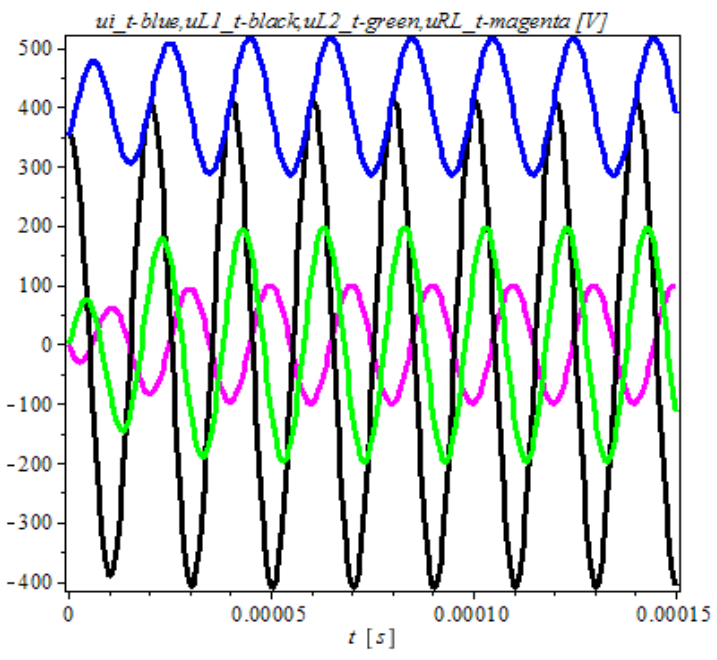

Figure 7. $u_{i_{-} t}, u_{L_{1} \_}, u_{L_{2} \_}$and $u_{\text {RL_t }}$ vs. time

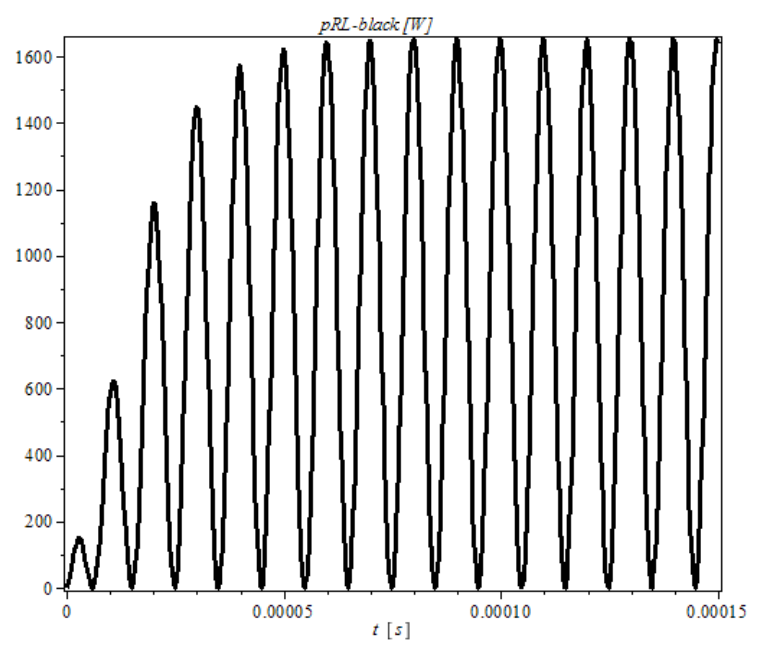

Figure 8. Load power $p_{\text {RL }}$ vs. time.

In Figure 9 we analyze the circuit form Figure 5 in frequency domain. Figure 9 depicts the load power $\left(P_{\mathrm{RL}}\right)$, the input power $\left(P_{1}\right)$ and efficiency $\eta_{21}$ function of frequency.

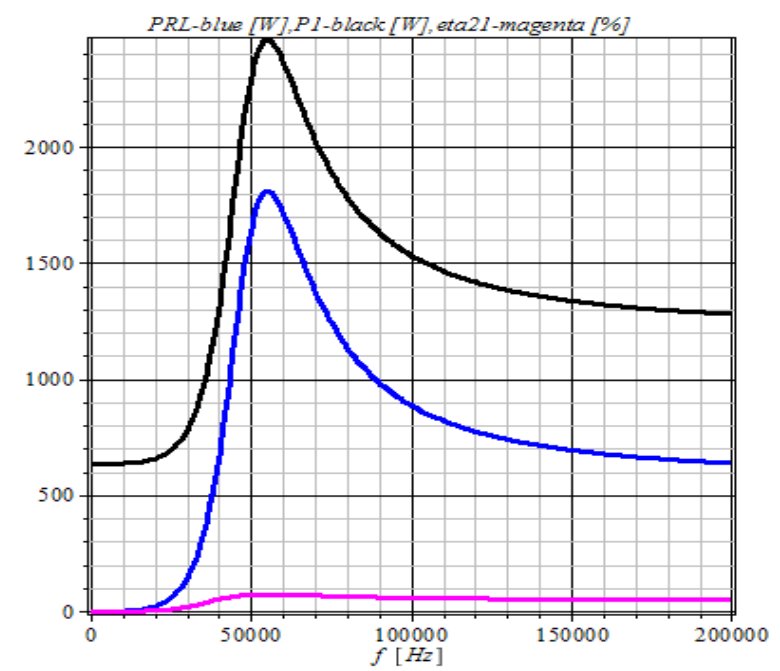

Figure 9. $P_{\mathrm{RL}}, P_{1,} \eta_{21}=100 * P_{\mathrm{RL}} / P_{1}$ function of frequency
Table 2 shows the maximum values of $P_{R L}$ and the $\eta 21$ with respect to frequency.

Table 2 Load power and efficiency function on frequency

\begin{tabular}{|c|c|c|}
\hline $\mathrm{f}[\mathrm{kHz}]$ & $\mathrm{P}_{\mathrm{RL}}[\mathrm{kW}]$ & $\eta_{21}[\%]$ \\
\hline$f_{0}=50.1$ & $\mathrm{P}_{\mathrm{RL} \_\mathrm{f} 0}=1.65025$ & $\eta_{21 \_f 0}=71.68$ \\
\hline $\begin{array}{c}f_{\text {PRLmax }}= \\
55.132207\end{array}$ & $\begin{array}{c}P_{\text {RL_fPRLmax }}= \\
1.8077262\end{array}$ & $\begin{array}{c}\eta_{21 \_f P R L m a x}= \\
73.450565\end{array}$ \\
\hline $\begin{array}{c}f_{\text {eta } 21 \max }= \\
55.1322\end{array}$ & $\begin{array}{c}P_{\text {RL_feta } 21 \max }= \\
1.8077279\end{array}$ & $\begin{array}{c}\eta_{21 \_f e t a 21 \max }= \\
73.450613\end{array}$ \\
\hline
\end{tabular}

where:

- $P_{\mathrm{RL} \text { feta21max }}\left(\eta_{21 \_ \text {fPRLmax }}\right)$ is the load power (efficiency) for the that frequency which ensures the maximum value of the efficiency (load power).

\section{CONCLUSIONS}

ANSYS Q3D Extractor tool, using the quasi-static solvers (method of moments, integral equations and FEMs), is very efficient for computing and simulating two- and three-dimensional electromagnetic field. This simulation is used to extract parameters of interest, such as: resistance $(\mathrm{R})$, conductance (G), self-inductance (L), mutual inductance $(\mathrm{M})$ and capacitance $(\mathrm{C})$. These parameters can be further used to optimize and to obtain automatically the design of a wireless power transfer systems (WPTS).

The resulting matrices allow the generation of new matrices for any selected parameters. To complete the analysis, the model which is computed will be exported as an equivalent circuit. In this paper, starting from a given WPTS, using ANSYS Q3D Extractor tool, the parameters of interest (R, G, L, M, C) were automatically calculated, and the SPICE equivalent circuit was obtained. The analysis was performed for two different distances between the emitter and receiver $(\mathrm{z}=20.2 \mathrm{~mm}$ and $\mathrm{z}=$ $70 \mathrm{~mm}$ respectively).

We also used electrical circuit theory in the system analysis of magnetically coupled coil system. Starting from the parameters extracted by the ANSYS Q3D Extractor tool, the performance of the WPTS has been analysed both in time and frequency domain.

\section{ACKNOWLEDGMENTS}

The work was supported by the Operational Programme Human Capital of the Ministry of European Funds through the Financial Agreement 51675/09.07.2019, SMIS code 125125 .

\section{REFERENCES}

[1] Ansoft Q3D Extractor, User Guide, www.ansoft.com.

[2] http://www.ansys.com/Products/Simulation+Technol ogy/Systems+\&+Embedded+Soft whare/ANSYS+Simplorer.

[3] A. Karalis, J.D. Joannopoulos, M. Soljačić, "Efficient wireless non-radiative mid-range energy transfer", Annals of Physics, Vol. 323, January 2008, pp. 34-48.

[4] J. I. Agbinya (Editor), Wireless Power Transfer, River 
Publishers Series in Communications, Denmark, 2012, ISBN: 978-87-92329-23.

[5] Ki Young Kim, Wireless Power Transfer - Principles and Engineering Explorations, Published by InTech Janeza Trdine 9, 51000 Rijeka, Croatia, 2011.

[6] D. Niculae, M. Iordache, Lucia Dumitriu, "Magnetic coupling analysis in wireless transfer energy", The 7th International Symposium on Advanced Topics in Electrical Engineering, 2011, Bucharest, 12-14 May 2011, IEEE Xplore, pp.1-4.

[7] Ariseidis Karalis, J.D. Joannopoulos, Marin Soljacic, Efficient wireless non-radiative mid-range energy transfer, MIT 2006.

[8] J.A.Ricano, H. Rodriguez, H. Vasquez, "Experiment About Wireless Energy Transfer", 1-st International congress on instrumentation and applied sciences, Cancun, Mexico, October 2010, pp. 12-14.

[9] Robert A. Moffatt, Wireless Transfer of electric power, Thesis for Bachelor of Science in Physics under the supervision of Marin Soljačić, June 2009.

[10] M. Iordache, L. Mandache, D. Niculae, Lavinia Bobaru (Iordache), "On exact circuit analysis of frequency splitting and bifurcation phenomena in wireless power transfer systems," Proc. of the International Symposium on Signals, Circuits and Systems - ISSCS 2015, Iasi, Romania, 2015, pp. 2427.

[11]Lavinia Iordache (Bobaru), Mihai Iordache, „Optimization of Magnetic Coupled Resonator Structure Used in Wireless Electromagnetic Energy Transfer", U.P.B. Scientific Bulletin Series C Electrical Engineering, Vol 78. Iss. 4. 2016, ISSN 2286-3540, pp. 137-148.

[12] Theodor Tanase, Marius Teme, Stefan Oncescu, Mihai Iordache, Dragos Niculae, Lavinia Iordache, Victor Bucata, "Some Aspects Regarding Optimization of Wireless Power Transfer Transmission", Publisher: IEEE, IEEE Xplore, 2016 International Conference on Applied and Theoretical Electricity (ICATE), DOI: 10.1109/ICATE.2016.7754705, Page(s): 1 - 6.

[13] Dragos Niculae, Mihai Iordache, Marilena Stanculescu; Lavinia Iordache," The influence of the alignment between receiver and transmitter coils on efficiency of wireless transfer energy", IEEE Xplore, 2016 International Conference on Applied and Theoretical Electricity, DOI: 10.1109/ICATE.2016.7754704, Page(s): 1 - 5.

[14] Marilena Stănculescu, M. Iordache, D. Niculae, Lavinia Iordache, V. Bucată, „S Parameter Computation and Their Use for Electromagnetic Energy Wireless Transmission," IJCT - International Journal of Computers \& Technologies, Vol. 12, No, June 2016, pp. 37097-7108.

[15] Maria-Lavinia Iordache (Bobaru), "Optimization of magnetically coupled resonators used in wireless power transfer", The fundamental field of Engineering Sciences - Electrical Engineering field, Faculty of Electrical Engineering, Department of Electrical Engineering, Bucharest, Doctoral Degree awarded under the Ministerial Order Education, Research, Youth and Sports No. 626 of 04.06.2018.

[16] Theodor Tănase, Marius Teme, Ștefan Oncescu, Mihai Iordache, Dragoș Niculae, Maria - Lavinia Iordache(Bobaru), Victor Bucată. „Some Aspects Regarding Optimization of Wireless Power Transfer Transmission" Proceeding of International Conference on Applied and Theoretical Electricity University of Craiova, Craiova ICATE 2016, 6 - 8, Otombrie, WOS:000390767500103, ISBN:978-14673-8562-6

[17] Mihai Iordache, Ioana-Gabriela Sirbu, Lucian Mandache, Andrei Marinescu, Dragos Niculae, Maria - Lavinia Iordache(Bobaru), "Comparative study of the frequency splitting and bifurcation phenomena for equivalent circuits of the wireless power transfer system”,IEEE Xplore, 2016 International Conference on Applied and Theoretical Electricity (ICATE), Page(s): 1 - 7. WOS:000390767500100, ISBN:978-14673-8562-6, ISSN: 2376-4163

[18] Mihai Iordache, George Andronescu, Victor Bucată, Dragoș Niculae, Marilena Stănculescu, Maria Lavinia Iordache(Bobaru), „Analysis and design of wireless electromagnetic energy transfer systems", Proceeding of International Conference on Applied and Theoretical Electricity - Craiova ICATE 2014, 68Otombrie, $\quad$ pp.8 - 16. DOI: 10.1109/ICATE.2016.7754703. 